\title{
Availability of Standard Operating Guidelines for Primary Healthcare Facilities in Sub-saharan Africa: A Scoping Review Protocol
}

Nelly Sharpley ( $\nabla$ nelicious2@gmail.com )

Faculty of Humanities, Social Sciences and Law, Walter Sisulu University, Nelson Mandela Drive, Private Bag X1, Mthatha, 5117, Eastern Cape, South Africa https://orcid.org/0000-0001-7657-4033

Desmond Kuupiel

University of Kwazulu-Natal

Jacqueline Marina van Wyk

University of Kwazulu-Natal

\section{Protocol}

Keywords: standard-operating guidelines, standard operating procedures, Primary Healthcare clinics, Community-based healthcare, Sub-Saharan Africa

Posted Date: December 18th, 2020

DOl: https://doi.org/10.21203/rs.3.rs-128292/v1

License: (c) (i) This work is licensed under a Creative Commons Attribution 4.0 International License. Read Full License 


\section{Abstract}

Background: Primary healthcare (PHC) clinics play an essential role in the delivery of community-based health services, particularly in resource-limited settings. However, there is little systematic documentation on the availability of standard operating guidelines governing their operation or evaluating the services provided by PHC facilities. This study, therefore, will aim to systematically map literature and describe the evidence on the availability of standard operating guidelines or procedures for PHC facilities in subSaharan Africa (SSA).

Methods: This scoping review study will be conducted inline with the Arksey and O'Malley's framework. PubMed, EBSCOhost (Academic search complete, CINAHL with full text, and Health Sources), Scopus, and Google Scholar will be searched from 2010 to 2020 for relevant evidence sources using a search strategy comprising of keywords, Boolean terms, and medical subject headings. This study will include evidence sources/studies written/published in English that focused standard on standard operating guidelines for staffing and operational management, patient care and service provision, and quality monitoring procedures to assess the functioning of PHC facilities in SSA. The screening of the evidence sources/studies at all the stages and the data extraction will be conducted independently by two reviewers. Thematic analysis will be carried out to identify the themes and sub-themes and the findings relating to this study's research question summarised and reported for each theme narratively.

Discussion: This scoping review anticipates to highlight standard operating guidelines available for staffing and operational management, patient care and service provision, and quality monitoring procedures to assess the functioning of $\mathrm{PHC}$ facilities to inform the development of relevant standard operating guidelines to improve healthcare services at the primary level. This study also anticipates revealing literature gaps for future research to ensure the attainment of universal health coverage by 2030.

\section{Background}

An essential component to achieve the United Nations sustainable development goals is the implementation of universal health coverage (UHC). However, ensuring the availability and accessibility of healthcare to all in Sub-Saharan Africa (SSA) like elsewhere in the world remains challenging [1, 2]. Despite the African region being burdened by about $24 \%$ of the global burden of disease [2, 3], it is severely challenged to ensure equitable health care services at the community level. This challenge is a result of a frail health system (weak governance), lack of human resources, limited financial resources, unreliable supply chains, and increasing populations, high burden of communicable and noncommunicable diseases coupled with poorly developed health infrastructure in several SSA countries [3, 4].

A 2017 report by the World Bank indicates that at least half the world's population still lacks access to essential health services [5]. With reference to UHC services, the report identified SSA as having the lowest 
coverage index value (42) compared to Southern Asia (53), East Asia (77) and Northern America and Europe (77) [5]. Community-based health services have been implemented to address the shortage and to improve healthcare coverage [2]. Community-based health services may comprise of preventive, promotive, curative services, and rehabilitation depending on the country's disease burden, the gap in service coverage, and the availability of funding [2]. The competencies of Community health workers vary greatly, and they deliver services related to sexual and reproductive health, tuberculosis, human immunodeficiency virus, disease surveillance and contact tracing, mental healthcare, and others to individuals, families, and communities $[2,6]$.

Community-based services such as those offered in clinics and health centres enhance equitable access to healthcare at the primary level [8]. Such services play a vital role to prevent the spread of disease, and effective and prompt treatment can provide holistic care. It is therefore essential that community-based primary healthcare (PHC) systems be strengthened [7]; especially in resource-limited settings where people face numerous challenges. The challenges include inadequate health infrastructure, insufficient staffing with high attrition, low referral systems, lack of standardised practice, poor planning for medication and medical supplies and inadequate supervision $[8,9]$.

The success and sustainability of community-based health services thus rest on the uniform application of standard guidelines and adherence to operational procedures. Whilst there are abundant publications on the elements of community-based work and types of services being provided, there is little systematic documentation on the availability and use of standard operational guidelines to evaluate the services provided by community-based health facilities. This scoping review aims to systematically map literature and describe the evidence on the availability of standard operating guidelines or procedures for $\mathrm{PHC}$ facilities in SSA focusing on standard operating guidelines for staffing and operational management, patient care and service provision, and quality monitoring procedures to assess the functioning of $\mathrm{PHC}$ facilities in SSA. Findings from this study will help to address governance challenges at the PHC level, improve community-based healthcare services towards the attainment of UHC, and it will help to identify gaps for future research in SSA.

\section{Methods}

This study will be a scoping review. Findings will enable us to search and select literature systematically, and synthesised the available evidence on the standard operational guidelines/policies for communitybased health facilities in SSA $[10,11]$. Aside from this, the scoping review method will enable us to map the concepts and describe the range of evidence available and identify research gaps $[10,11]$. This scoping review will be guided by an adapted Arksey and O'Malley framework [10,12]. The framework comprised of the following: identifying the review question; identifying relevant evidence sources/studies; study selection; charting the data; and collating, summarising and reporting the results. To aid the development of this study protocol, we adopted the preferred reporting items for systematic review and meta-analysis for protocols (PRISMA-P), but the resulting paper will be reported in line with the PRISMA extension for a scoping review checklist [13]. 


\section{Identifying the research question}

The main research question this scoping review will seek to address is: To date, what evidence exists on the availability of standard operating guidelines or procedures for PHC facilities in SSA?. The sub-review questions are as follows:

1. What standard operating guidelines exist for staffing and operational management of PHC clinics in SSA?

2. What standard operating guidelines exist for patient care and service provision at PHC clinics in SSA?

3. What standard operating guidelines exist for quality monitoring procedures to assess the functioning of PHC facilities in SSA?

\section{Identifying relevant evidence sources}

Searches will be conducted on PubMed, EBSCOhost (Academic search complete, CINAHL with full text, and Health Sources), Scopus, and Google Scholar from 2010 to 2020 for relevant evidence sources to answer the review question. The search will employ keywords, Boolean terms ("AND" and "OR"), and medical subject headings to develop a comprehensive search strategy (Table 1). The syntax will be modified where needed. The team will also use the services of an experienced subject librarian to ensure that a robust review search strategy is followed. The PCC framework helped to specify the scope of the relevant publications.

Limitations on study design will be removed, but language limitations will be included where possible during the search. The search records will be documented appropriately. That is search date, database, keywords, search results, and count of eligible articles. To guide the electronic search strategy, the PRESS (Peer Review of Electronic Search Strategies) statement will be used [14]. The reference list of the included sources of evidence, Google, and the WHO website will also be consulted for evidence relating to standard operating guidelines for PHC level facilities in SSA. To compile all relevant evidence sources, identify and remove duplicate records, we will use the EndNote X9 reference manager. NS will search for the evidence sources assisted by the review team and import them onto an EndNote library created for this review.

Table 1: Pilot search in PubMed electronic database 


\begin{tabular}{|c|c|c|}
\hline Date & Keywords & $\begin{array}{l}\text { Search } \\
\text { results }\end{array}$ \\
\hline $07 / 12 / 2020$ & 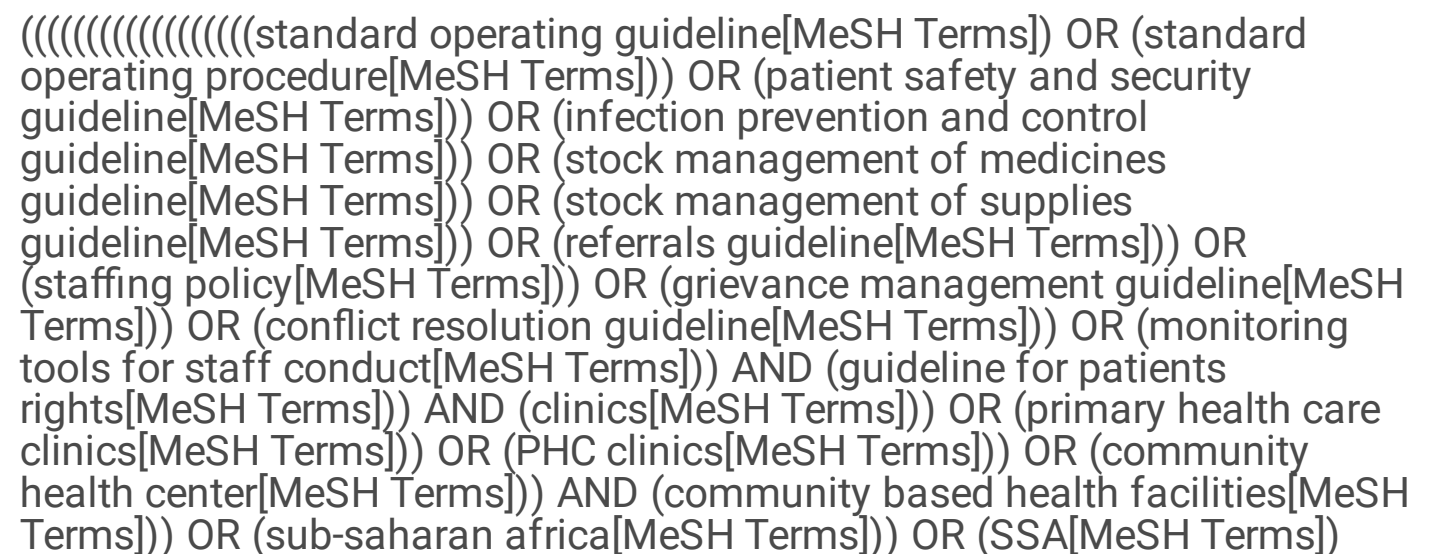 & 4,524 \\
\hline
\end{tabular}

\section{Study/evidence source selection}

\section{Eligibility criteria}

Table 2 below summarises this scoping review's eligibility criteria.

Table 2: Eligibility criteria for selection of an evidence source 


\begin{tabular}{|c|c|c|}
\hline $\begin{array}{l}\text { Eligibility } \\
\text { criteria }\end{array}$ & Inclusion criteria & $\begin{array}{l}\text { Exclusion } \\
\text { criteria }\end{array}$ \\
\hline Population & PHC clinics or clinics or community health centres & Hospitals \\
\hline Concept & $\begin{array}{l}\text { Standard operating guidelines or procedures for patient safety and } \\
\text { security or infection prevention and control, or stock management of } \\
\text { medicines and supplies, or patient referrals, or staffing policy, or } \\
\text { grievance management or conflict resolution or monitoring tools for } \\
\text { staff conduct or observation of patients rights. }\end{array}$ & \\
\hline Context & SSA countries & $\begin{array}{l}\text { Publication on } \\
\text { countries } \\
\text { from outside } \\
\text { the WHO } \\
\text { Africa Region }\end{array}$ \\
\hline $\begin{array}{l}\text { Publication } \\
\text { status }\end{array}$ & $\begin{array}{l}\text { Published papers, guidelines/policies unpublished articles, or grey } \\
\text { literature }\end{array}$ & \\
\hline Language & English & $\begin{array}{l}\text { Articles that } \\
\text { are written in } \\
\text { French, } \\
\text { Portuguese, } \\
\text { Arabic, other } \\
\text { local } \\
\text { languages }\end{array}$ \\
\hline $\begin{array}{l}\text { Study } \\
\text { designs }\end{array}$ & All study designs & \\
\hline $\begin{array}{l}\text { Publication } \\
\text { during a } \\
\text { specific } \\
\text { period }\end{array}$ & 2010 to 2020 & $\begin{array}{l}\text { Publications } \\
\text { before } 2010\end{array}$ \\
\hline
\end{tabular}

\section{Selection of evidence source}

Following the compilation of all evidence sources, the EndNote library will be cleaned by deleting all duplicate records. All tools or forms that will be used for the selection of the evidence sources will be piloted tested, and the needed amendments made to ensure their accuracy and reliability. Subsequently, the EndNote library will be shared among the review team. To reduce selection bias, two reviewers (NS and JvW) will conduct the title and abstract, and the full-text article screening independently. Guided by this scoping review eligibility criteria, the evidence sources will be sorted into either "include" or "exclude" group. At the abstract stage, discrepancies that may arise between NS and JvW will be discussed by the review team until a consensus is reached. A third reviewer will independently address any discrepancies that ensue between NS and JvW at the full text screening stage. We will use the University of KwaZuluNatal library services to retrieve all full-text articles with closed access publications. Emails will also be sent to the original authors to request relevant full-text articles if needed. The various stages of the 
evidence sources selection will be appropriately documented using an amended PRISMA flow diagram (Figure 1) [15].

\section{Charting the Data}

A spreadsheet will be developed for the data charting. The spreadsheet will be piloted independently by NS and DK using a minimum of ten percent of the included evidence sources to ensure consistency. The review team will resolve all discrepancies, and the needed amendments will be done before its final usage to enable the capturing of all relevant data and reliability of this study results. The data will be extracted using both inductive and deductive approaches [16]. The following data will be collected:

1. Author, publication year

2. Title of publication

3. Aims/objective of the publication

4. The geographical location (Country)

5. Type of evidence (grey literature/unpublished/peer-review publication)

6. Study design (where applicable)

7. Study participants (where applicable)

8. Type of standard operating guideline/procedure

9. Type of PHC facility

10. Findings relating to standard operating guideline/procedure/patient management at the PHC level 11. Relevant conclusion and recommendations.

\section{Collating, Summarising, and Reporting the Results}

The data extracted will be analyzed using thematic content analysis approach (coding and categorisation) [16]. Relevant findings for each of the themes or sub-themes will be summarised and reported narratively. Figures and tables where appropriate will be used to present the characteristics of the evidence sources.

\section{Discussion}

This scoping review study aims to systematically map literature and describe the evidence on the availability of standard operating guidelines/procedures for PHC facilities in SSA. The world has set out to attain universal health coverage by 2030 , and the role of PHC clinics services is highly essential. To this end, systematic documentation on the availability of standard operating guidelines governing PHC clinic operations or use to evaluating the services provided by PHC facilities is much needed. Given this, this study will source the most recent literature published within the last ten years relating to standard operating guidelines/procedures for PHC facilities in SSA focusing on staffing and operational management, patient care and service provision, and quality monitoring procedures to assess the 
functioning of PHC facilities. Due to lack of expertise in other official languages, this study will include only literature published in English. This study anticipates revealing evidence to inform the development of relevant standard operating guidelines to improve healthcare services at the primary level. This study also anticipates revealing literature gaps for future research to ensure the attainment of universal health coverage by 2030 .

\section{List Of Abbreviations}

PHC: Primary Healthcare

SSA: Sub-Saharan Africa

UHC: Universal Health Coverage

WHO: World Health Organisation

\section{Declarations}

\section{Conflict of Interest}

None reported

\section{Ethics approval}

Not applicable. All sources used have been appropriately cited and included in the reference list.

Consent for Publication

N/A

\section{Availability of data and materials}

N/A

\section{Competing Interests}

The authors declare no competing interests.

\section{Funding}

$\mathrm{N} / \mathrm{A}$

\section{Author's Contributions}

NS conceptualised the study under the supervision of JvW. DK designed the study methodology. NS wrote the manuscript, and DK and JvW critically reviewed it and made revisions. All authors read and 
approved the final version of the manuscript.

\section{Acknowledgement}

None

\section{References}

1. Organization WH. Declaration of Alma-Ata. 2000.

2. World Health Organization. Community-based Health Services: a vital part of Universal Health Coverage Geneva: World Health Organization; 2016 [cited 2020 22/11/2020]. Universal Health Coverage discussion paper 1: 2016:[Available from https://apps.who.int/iris/rest/bitstreams/1167216/retrieve.

3. Lehmann U, Twum-Danso NAY, Nyoni J. Towards universal health coverage: what are the system requirements for effective large-scale community health worker programmes? BMJ Global Health. 2019;4(Suppl 9):e001046.

4. Kuupiel D, Bawontuo V, Mashamba-Thompson TP. Improving the Accessibility and Efficiency of Point-of-Care Diagnostics Services in Low- and Middle-Income Countries: Lean and Agile Supply Chain Management. Diagnostics (Basel). 2017;7(4):58.

5. World Health Organization, Reconstruction IBf, and Development, The World Bank. Tracking Universal Health Coverage: 2017 Global Monitoring Report Geneva: World Health Organization; 2017 [cited 2020 22/11/2020]. Available from: http://pubdocs.worldbank.org/en/193371513169798347/2017-global-monitoring-report.pdf.

6. Covert H, Sherman M, Miner K, Lichtveld M. Core Competencies and a Workforce Framework for Community Health Workers: A Model for Advancing the Profession. Am J Public Health. 2019;109(2):320-7.

7. De Maeseneer J, Li D, Palsdottir B, Mash B, Aarendonk D, Stavdal A, et al. Universal health coverage and primary health care: the 30 by 2030 campaign. Bull World Health Organ. 2020;98(11):812-4.

8. Assan A, Takian A, Aikins M, Akbarisari A. Challenges to achieving universal health coverage through community-based health planning and services delivery approach: a qualitative study in Ghana. BMJ Open. 2019;9(2):e024845.

9. Kuupiel D, Tlou B, Bawontuo V, Drain PK, Mashamba-Thompson TP. Poor supply chain management and stock-outs of point-of-care diagnostic tests in Upper East Region's primary healthcare clinics, Ghana. PLoS One. 2019;14(2):e0211498-e.

10. Arksey H, O'Malley L. Scoping studies: towards a methodological framework. International journal of social research methodology. 2005;8(1):19-32.

11. Colquhoun HL, Levac D, O'Brien KK, Straus S, Tricco AC, Perrier L, et al. Scoping reviews: time for clarity in definition, methods, and reporting. Journal of clinical epidemiology. 2014;67(12):1291-4. 
12. Levac D, Colquhoun H, O'Brien KK. Scoping studies: advancing the methodology. Implementation Science. 2010;5(1):69.

13. Tricco AC, Lillie E, Zarin W, O'Brien KK, Colquhoun H, Levac D, et al. PRISMA Extension for Scoping Reviews (PRISMA-ScR): Checklist and Explanation. Annals of internal medicine. 2018;169(7):467-73.

14. McGowan J, Sampson M, Salzwedel DM, Cogo E, Foerster V, Lefebvre C. PRESS Peer Review of Electronic Search Strategies: 2015 Guideline Statement. Journal of Clinical Epidemiology. 2016;75:40-6.

15. Moher D, Liberati A, Tetzlaff J, Altman DG, The PRISMA Group. PRISMA 2009 Flow Diagram. 2009;6(2009):1000097.

16. Fereday J, Muir-Cochrane E. Demonstrating rigor using thematic analysis: A hybrid approach of inductive and deductive coding and theme development. International journal of qualitative methods. 2006;5(1):80-92.

\section{Figures}




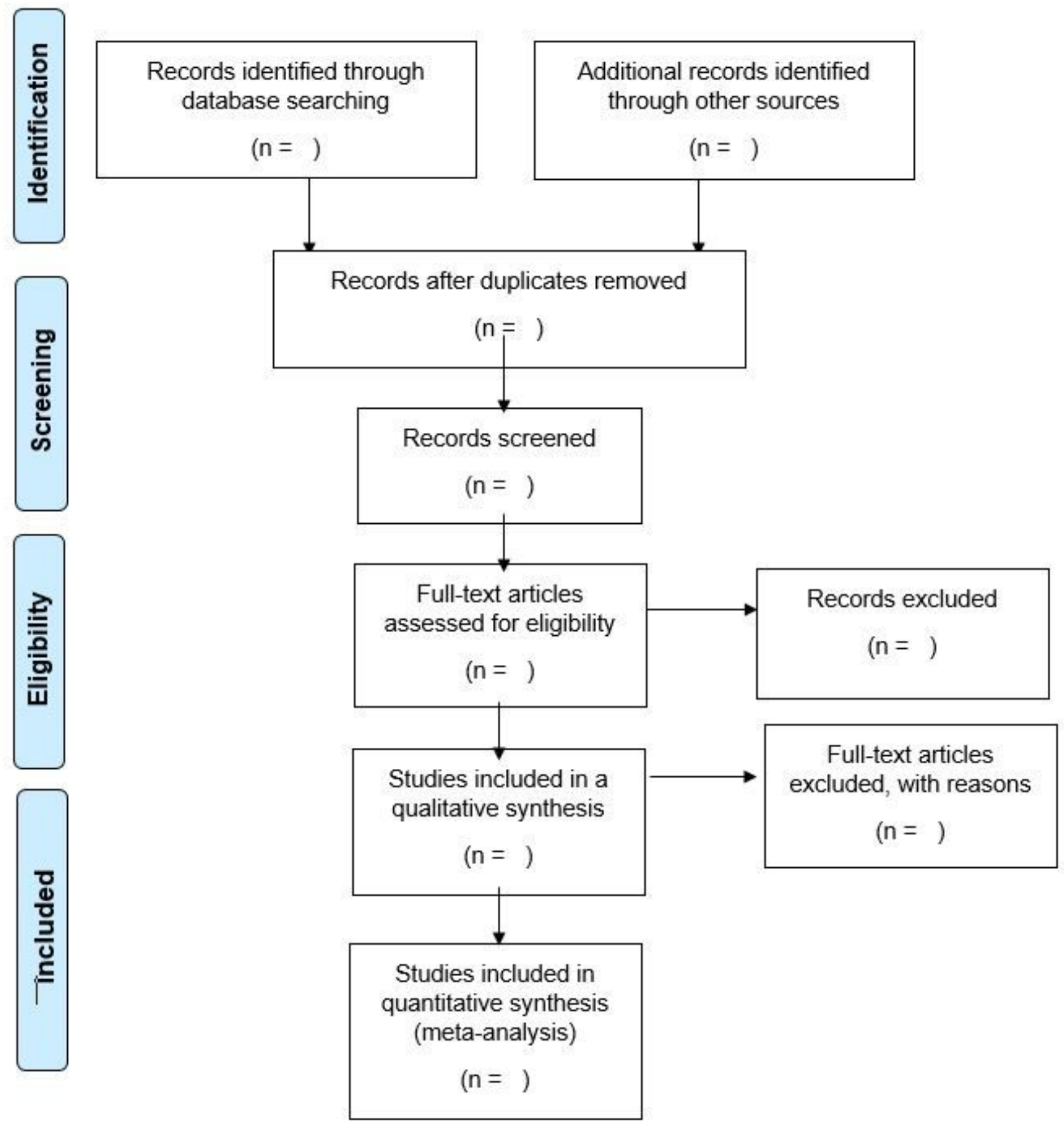

Figure 1

PRISMA 2009 Flow Diagram [15] 


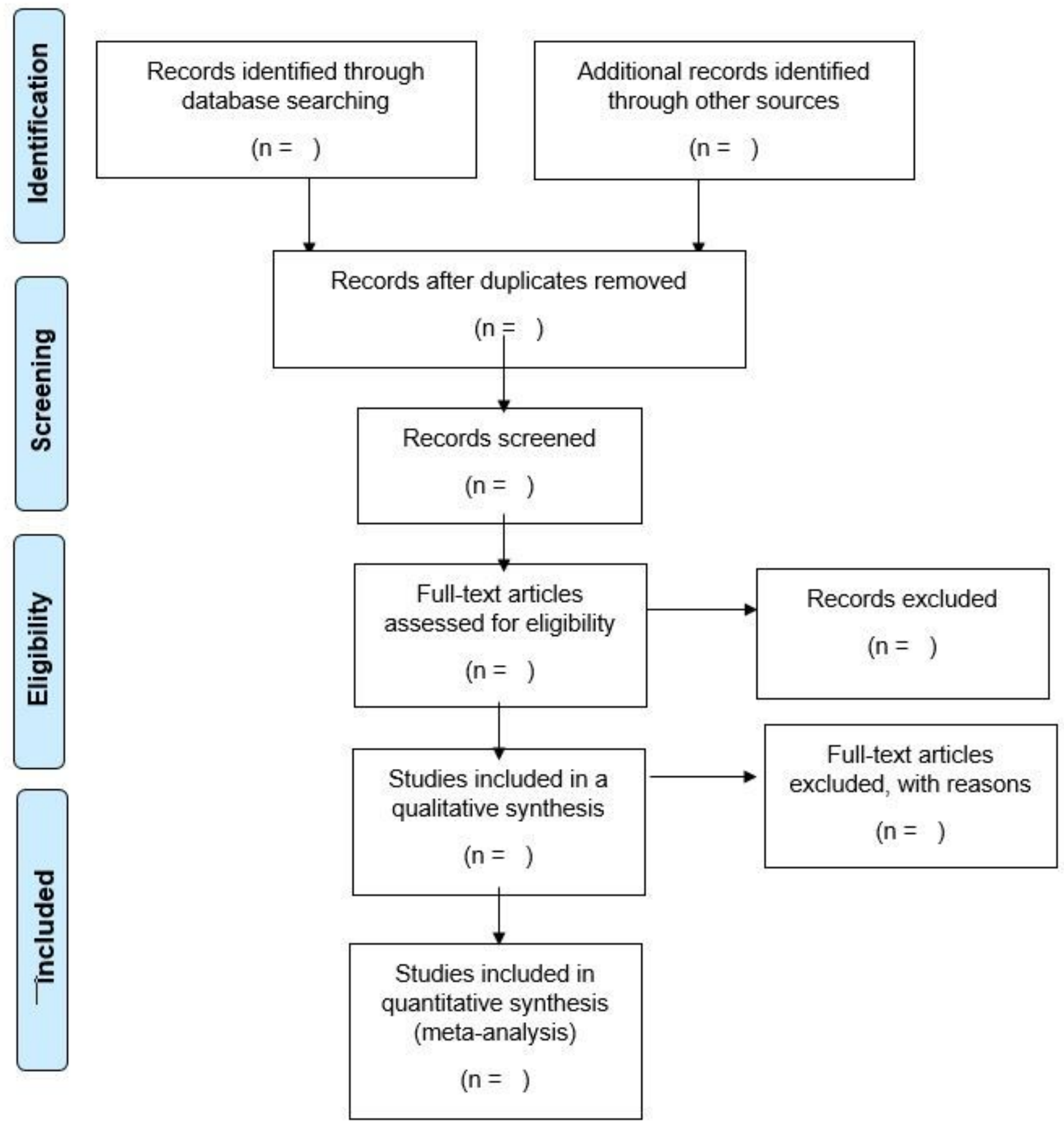

Figure 1

PRISMA 2009 Flow Diagram [15]

\section{Supplementary Files}

This is a list of supplementary files associated with this preprint. Click to download. 
- PRISMPchecklistbyDrSharpley.docx

- PRISMPchecklistbyDrSharpley.docx 